\title{
Performance and Attributes of Liquid Chromatography-Mass Spectrometry with Targeted Charge Separation in Quantitative Analysis of Therapeutic Peptides
}

\author{
Changtong Hao, ${ }^{1}$ J. Larry Campbell, ${ }^{2}$ Udo H. Verkerk, ${ }^{1}$ J. C. Yves Le Blanc, ${ }^{2}$ \\ K. W. Michael Siu ${ }^{1}$ \\ ${ }^{1}$ Department of Chemistry and Centre for Research in Mass Spectrometry, York University, 4700 Keele Street, Toronto, ON \\ M3J 1P3, Canada \\ ${ }^{2}$ AB SCIEX, 71 Four Valley Drive, Concord, ON L4K 4V8, Canada
}

\begin{abstract}
Herein we describe a new method, targeted enhanced multiply charged scans (tEMC), for the quantification of therapeutic peptides in tandem mass spectrometry on the linear ion trap mass spectrometer. Therapeutic peptides with chain lengths between eight and 39 amino acid residues and charge states from $2+$ to $6+$ were used to evaluate and illustrate the method which relies on the ability to separate ions trapped in a linear ion trap according to their charges. In particular, interference from singly charged ions on multiply charged ions can be effectively minimized. The method requires optimization of relatively few parameters, the most important of which being the exit lens barrier (EXB) voltage, thereby offering substantial time saving in a high-throughput quantification environment that currently relies on selected reaction monitoring.
\end{abstract}

Key words: Quantification, Therapeutic peptides, Linear ion trap mass spectrometry, High-throughput quantification, Selected reaction monitoring, Trapped ion charge discrimination

\section{Introduction}

$\mathrm{P}$ roteins and peptides are gaining importance as therapeutic drugs $[1,2]$ owing to their high activity, specificity, and low toxicity $[3,4]$. With this increased interest, it is becoming necessary to rapidly develop quantitative methods for therapeutic peptides that will support a dynamic drug-discovery environment. Liquid

Electronic supplementary material The online version of this article (doi:10.1007/s13361-010-0015-6) contains supplementary material, which is available to authorized users.

Correspondence to: J. C. Yves Le Blanc; e-mail: yves.leblanc@sciex. com, K. W. Michael Siu; e-mail: kwmsiu@yorku.ca chromatography (LC) interfaced with tandem mass spectrometry (MS/MS) is one of the preferred analytical technologies, as it provides high sensitivity and selectivity when operated in multiple reaction monitoring (MRM) or, more generically, selected reaction monitoring (SRM) mode [5]. Though widely used in the pharmaceutical industry for assaying small-molecule drugs, SRM suffers from a number of limitations that hinder its application to peptide-based drugs, especially in a high-throughput environment-recent applications in the quantification of tryptic peptides in the analysis of blood plasma notwithstanding [6, 7].

Unlike small organic molecules, peptides have a large number of basic sites, many of which are protonated in the gas phase under standard electrospray conditions. The 
resulting cluster of charge states divides the analytical signal and decreases signal to noise; monitoring of several charge states and their associated fragments is typically required for unambiguous determination, which ultimately restricts the lower limit of quantification (LLOQ) [8]. While having a host of fragment ions is advantageous to peptide identification [9], extensive fragmentation of multiply charged peptides further adds to the time-consuming selection of optimal SRM transitions, especially in the presence of interferences due to a biological matrix $[10,11]$. Indeed, the process for optimizing SRM transitions for multiply charged peptides can become tedious and time-consuming, thus increasing the analytical development time. The MS equivalent of SRM in MS/MS is single ion monitoring (SIM). While SIM can provide greater sensitivity than SRM in terms of higher absolute ion signal intensity and better counting statistics, this procedure can only be satisfactorily applied to samples in simple matrices, such as neat solvents, and lacks the selectivity required for the analysis of biological extracts [10, 12].

As the chemical background in MS of complex biological samples comprises primarily singly charged ions, techniques that can discriminate multiply charged ions from singly charged ions (chemical noise) prior to MS detection have been developed. Classical ion mobility as well as differential mobility (in differential mobility spectrometry (DMS) and high-field asymmetric waveform ion mobility spectrometry (FAIMS) [13, 14]) have shown some degree of discrimination between charge states, but more importantly between singly charged ions and multiply charged ions as a group $[15,16]$. Consequently, ion mobility-based techniques coupled to MS-based detection, such as SIM or MRM, have been proposed and evaluated as technologies to improve the quantification of peptides [17-20]. Alternatively, multiply charged peptide ions can be separated from singly charged ions prior to MS detection in a procedure known as the enhanced multiply charged (EMC) scan [21-23]. During an EMC scan, a population of ions that can include singly and multiply charged species is contained within a linear ion trap (LIT) [22]. Typically, analysis of such a population will result in the multiply charged peptide ions buried in the sea of singly charged ions that constitutes the chemical background. Trapping of ions in the LIT necessitates the application of repulsive potentials to lenses that bookend the LIT [22]. The energy barrier experienced by the ions in
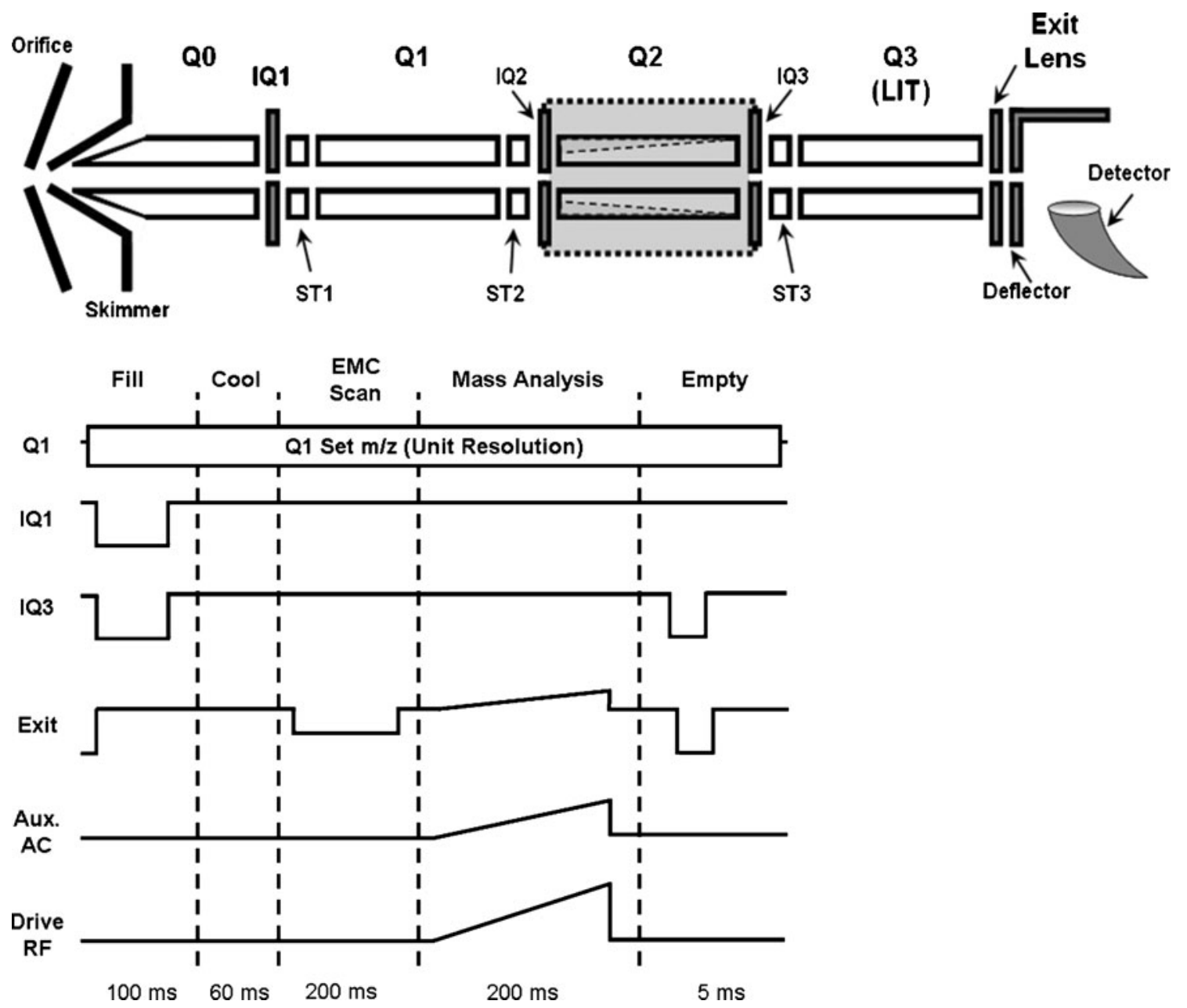

Figure 1. QTRAP 4000 mass spectrometer: upper panel, ion optics; lower panel, voltage timing diagram. ST, stubbies; Q, quadrupole rod set; IQ, interquad lens; LIT, linear ion trap 
the well is proportional to their charges: for a repulsive potential of $1 \mathrm{~V}$, singly charged ions will face an energy barrier of $1 \mathrm{eV}$, while doubly charged ions will experience a $2 \mathrm{eV}$ barrier. By controlling the d.c. potentials applied to these lenses, conditions can be established such that multiply charged ions are selectively retained, whereas singly charged ions escape over the lower barrier against which they are subjected [22]. By adding precursor ion selection with quadrupole $(\mathrm{Q} 1)$ set to mass-resolving mode prior to the EMC scan, selective detection of multiply charged ions can be achieved without the need for fragmentation and MS/MS. This extended mode of operation has been referred to as targeted enhanced multiply charged (tEMC) scan [24, 25].

In this study, we evaluate use of the tEMC scan mode for the quantification of multiply charged peptides on a hybrid quadrupole-LIT mass spectrometer. Comparisons of tEMC with traditional SRM-based approaches were performed to determine the precision, accuracy, LLOQs, and ease of use for a series of therapeutic peptides.

\section{Experimental}

\section{Materials}

Porcine dynorphin A; human angiotensin I; angiotensin II; arg-insulin; arg-vasopressin; bee melittin; exenatide; and ketoconazole (a synthetic pharmaceutical) were purchased from Sigma-Aldrich (Milwaukee, WI) and were used as received. HPLC-grade acetonitrile was purchased from JT Baker (Phillipsburg, NJ), and formic acid from EMD Chemicals (Gibbstown, NJ). Distilled deionized water was prepared in-house using a Milli-Q plus water purification system (Millipore, Bedford, MA).
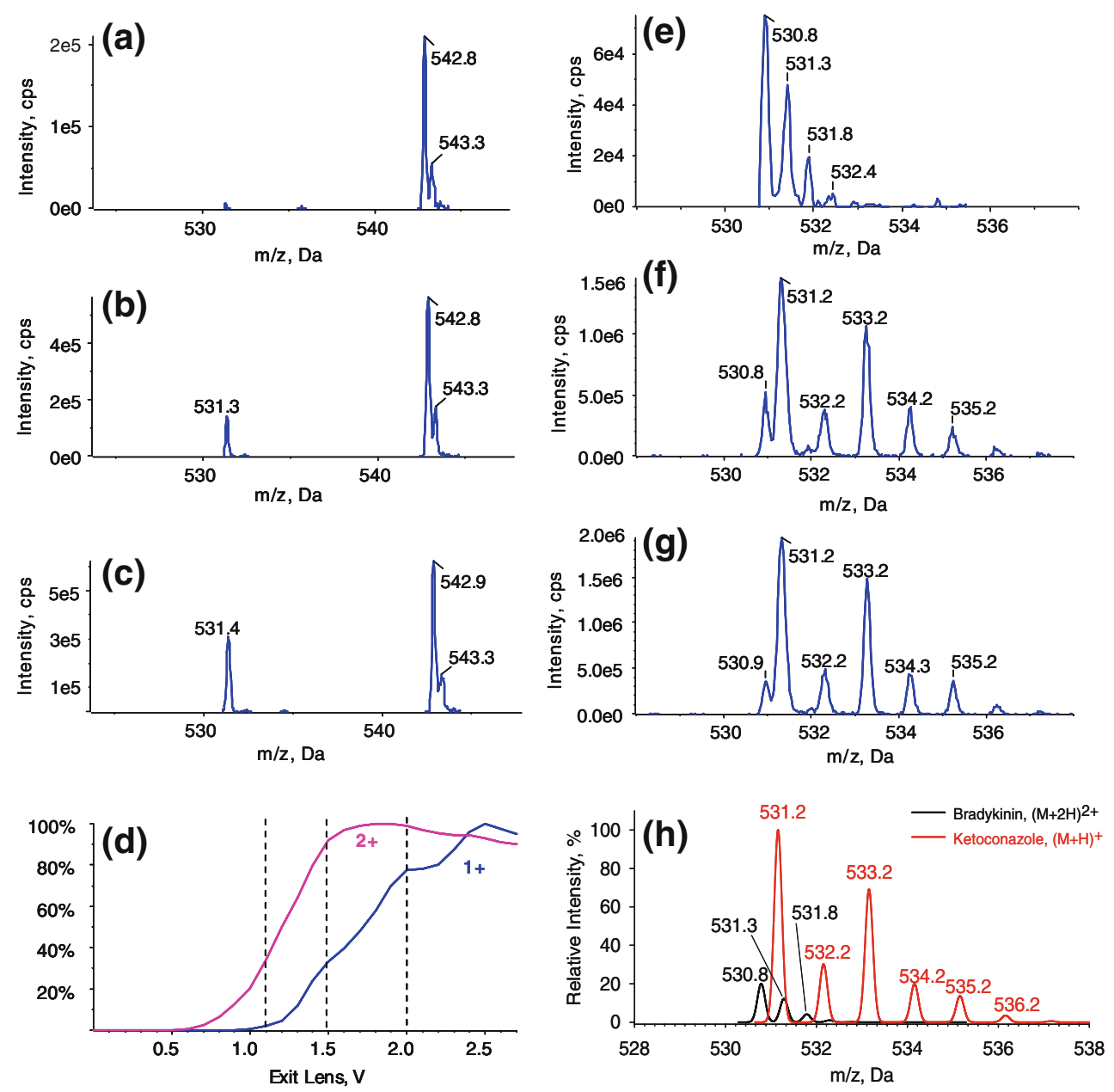

Figure 2. $\mathrm{tEMC}$ mass spectra (a-c) of a mixture of $0.1 \mathrm{pmol} / \mu \mathrm{L}$ ketoconazole (singly protonated at $\mathrm{m} / \mathrm{z} 531.3$ ) and $0.5 \mathrm{pmol} / \mu \mathrm{L}$ arg-vasopressin (doubly protonated at $\mathrm{m} / \mathrm{z} 542.8$ ) recorded at an exit lens voltage of (a) $1.1 \mathrm{~V}$, (b) $1.5 \mathrm{~V}$, and (c) $2.0 \mathrm{~V}$; (d) relative ion abundance of protonated ketoconazole and doubly protonated arg-vasopressin as a function of the exit lens voltage in $0.02-\mathrm{V}$ increments; tEMC mass spectra (e-g) of a mixture of $0.1 \mathrm{pmol} / \mu \mathrm{L}$ (singly protonated at $\mathrm{m} / \mathrm{z} 531.2$ ) and $1 \mathrm{pmol} /$ $\mu \mathrm{L}$ bradykinin (doubly protonated at $\mathrm{m} / \mathrm{z} 530.8$ ) with an exit lens voltage of (e) $1.1 \mathrm{~V}$, (f) $1.5 \mathrm{~V}$, and (g) $2.0 \mathrm{~V}$; (h) theoretical isotopic distribution of protonated ketoconazole and doubly protonated bradykinin at a ratio of 5:1 
Protein-precipitated horse plasma (Sigma-Aldrich) was used as biological matrix in the experiments. Use of this matrix minimized the risk of endogenous interference with the peptide selected, while still providing chemical interferences typically observed with biological samples. One volume of plasma was precipitated with two volumes of acetone, shaken for $5 \mathrm{~min}$, and centrifuged for $15 \mathrm{~min}$ at $9,000 \mathrm{rpm}(8,887 \mathrm{~g})$. The supernatant was diluted with twice the volume of water and was used as the solvent in subsequent preparations. This additional dilution step was added to lower the organic content of the solution and ensure proper loading of the peptide on column. For any given peptide, a stock solution of $5 \mathrm{pmol} / \mu \mathrm{L}$, stored at $-20{ }^{\circ} \mathrm{C}$, was serially diluted to give standard solutions covering the range from 1.22 to $2,500 \mathrm{fmol} / \mu \mathrm{L}$ used to construct the calibration curve. Serial dilution was deemed appropriate for preparation of samples in this particular study, as the relative performance of different MS-based techniques with the same instrument was at the core of the present study, and was independent of other experimental factors associated with the bioanalysis (e.g., extraction efficiency, sample handling, etc.). All analyses were performed in triplicates.

\section{Liquid Chromatography/Mass Spectrometry}

Peptide separations were conducted at room temperature using a POROS R2 $2.1 \times 100 \mathrm{~mm}, 10-\mu \mathrm{m}$ poly(styrenedivinylbenzene) column with a particle pore size of $2,000 \AA$ (Life Technologies, Carlsbad, CA) and at a flow rate of $500 \mu \mathrm{L} / \mathrm{min}$. A Perkin-Elmer series 200 (Norwalk, CT, USA) system that comprised an autosampler and two micropumps was employed for the LC separations. The solvents used as the mobile phase were A: 98:2 water/ acetonitrile $(\mathrm{v} / \mathrm{v})$ containing $0.2 \%(\mathrm{v})$ formic acid; and B: 98:2 acetonitrile/water ( $\mathrm{v} / \mathrm{v}$ ) also containing $0.2 \%$ (v) formic acid. Separation was performed by maintaining $98 \%$ of solvent A for $2 \mathrm{~min}$, a linear gradient to $40 \%$ A over $3 \mathrm{~min}$, followed by $2 \mathrm{~min}$ at $98 \% \mathrm{~B}$, and finishing with a 3-min column re-equilibration with $98 \%$ A before the next injection.

All mass spectrometry experiments were performed by using a 4000 QTRAP hybrid quadrupole-LIT mass spectrometer (AB SCIEX, Concord, Canada), a schematic of which is shown in Figure 1. For the SIM experiments, two of the most abundant ions (charge states) for each peptide were mass selected by Q1 (set to unit resolution) and transmitted to the detector with Q3 in r.f.-only mode. The dwell time for each of the ions was $300 \mathrm{~ms}$. The SRM experiments were conducted by monitoring two transitions for the two most dominant charge states of every peptide, with Q1 and Q3 operated at unit resolution. The tEMC data were acquired with Q1 at unit resolution and typically with the exit lens barrier (EXB) voltage optimized to provide optimal discrimination between multiply charged peptide ions and singly charged matrix ions.
Analyte-specific parameters employed in this study are given in the Supplementary Information section. The following parameters were common to all the experiments: curtain gas, $20 \mathrm{psi}$; TurboV Ionspray voltage, $+5 \mathrm{kV}$; ion source gas 1, $40 \mathrm{psi}$; ion source gas 2, $60 \mathrm{psi}$; source temperature, $400{ }^{\circ} \mathrm{C}$; and collision gas pressure, high. All experimental data were processed by using MultiQuant software 1.1 (AB SCIEX). Each peptide was analyzed in triplicate and the resulting calibration plot was constructed by linear regression with a weighting factor of $1 / x^{2}$. Each of the MS detection techniques was evaluated for linearity, accuracy (reported as the percent deviation from the mean), and precision (as the coefficient of variation (CV)). LLOQs were determined by using typical bioanalytical acceptance criteria in terms of accuracy and precision $(<20 \%$ for both) [26, 27].

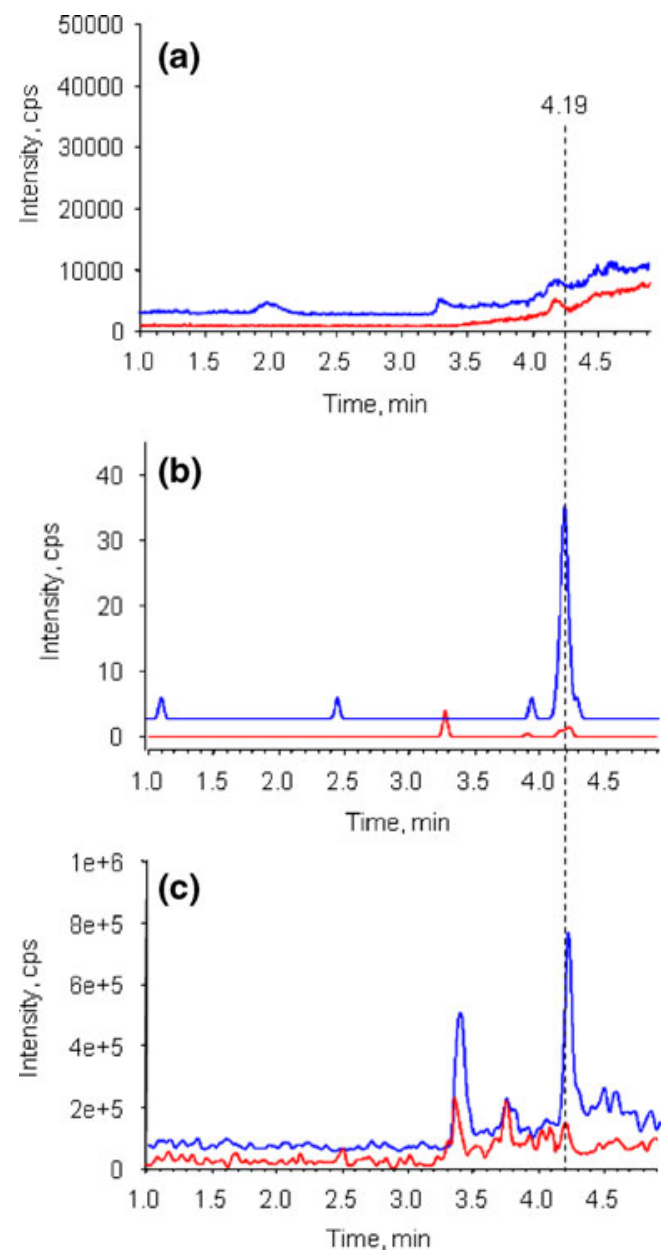

Figure 3. Liquid chromatograms of triply protonated angiotensin I ( $\mathrm{m} / \mathrm{z} 432.9), 12.3 \mathrm{fmol}$ in $10 \mu \mathrm{L}$ of dilute horse plasma, blue trace; horse plasma blank, red trace: (a) SIM; (b) SRM as sum of two transitions, $\mathrm{m} / \mathrm{z} 432.9$ to $\mathrm{m} / \mathrm{z} 619.8$ and 647.5 ; and (c) tEMC 


\section{Targeted Enhanced Multiply Charged Scans}

The tEMC scan utilizes the resolving power of Q1 to 'target' specific ions of interest for enhanced multiply charged scanning. By contrast, a standard EMC scan accepts all ions generated at the source and traps them in the Q3 LIT. In this study, Q1 was set to select ions at the predicted charge state(s) of the peptide analyte ions prior to charge separation. These ions were then transmitted to Q3, where they were trapped by the application of d.c. voltages on lens elements on both ends of Q3. After a short cooling time, the EXB voltage (applied to the exit lens that endcaps Q3, Figure 1) was lowered to allow singly charged ions to escape the LIT (vide supra), while multiply charged ions were retained. Following this step, the barrier was lowered and the ions scanned out by mass-selective axial ejection (MSAE) [28]. The analytical signal for the tEMC scan is an extracted ion chromatogram derived from the signal at the $\mathrm{m} / \mathrm{z}$ of the analyte ion with a window of $\pm 0.1 \mathrm{Th}$.

A timing diagram showing the relative voltages on key elements during various phases of the experiment is presented in Figure 1, lower panel. Optimal conditions for the tEMC experiment were: Q1 resolution, unit; Q3 scan window, 10-Th wide and centered on the $\mathrm{m} / \mathrm{z}$ of interest; fill time, $100 \mathrm{~ms}$; EMC scan time, $200 \mathrm{~ms}$; Q3 entrance barrier, $5 \mathrm{~V}$; CE, $5 \mathrm{~V}$; EXB, 0.8-1.1 V; declustering potential, compound specific.

\section{Results and Discussion}

\section{Establishing Optimal Conditions for tEMC}

The tEMC approach, which selectively enhances the detection of multiply charged analyte ions without using fragmentation, can be rapidly implemented on linear ion trap mass spectrometers. This is in contrast to methods that require fragmentation (e.g., SRM-based experiments), which often require considerable time and efforts to optimize fully. tEMC scans require the optimization of only a handful of parameters under the current implementation mode, including LIT fill time, EMC scan time, and the exit lens barrier or EXB. Similar to any linear ion trap scan and under ideal implementation of tEMC, the fill time would need to be adjusted dynamically based on the ion flux generated at the source. Under these conditions, when the signal intensity is weak (low analyte concentration), a longer fill time would be used to maximize sensitivity. By contrast, when the signal intensity is strong (high analyte concentration), a shorter fill time could be used. As dynamic control of the fill time was not available for tEMC scan, a fixed fill time of $100 \mathrm{~ms}$ was used for all experiments. To determine the EMC scan time (empty time), the trap was filled with both singly and doubly charged ions of different abundance ratios and the EMC scan time was varied from 50 to $300 \mathrm{~ms}$, in increments of $50 \mathrm{~ms}$. An EMC scan time of $200 \mathrm{~ms}$ was found to effectively discriminate at least $98 \%$ of singly charged ions, even when the trap contained singly charged ions in a 10-fold excess of doubly charged ions. Consequently, a fixed fill time and EMC scan time of $100 \mathrm{~ms}$ and $200 \mathrm{~ms}$, respectively, were used for all analytes examined. The parameter that bore the greatest influence on the performance on the tEMC scans was the EXB voltage which controlled the discrimination of singly charged, background ions.

To illustrate and evaluate the effect of EXB on discriminating ions of different charge, a mixture of two compounds, ketoconazole $(0.1 \mathrm{pmol} / \mu \mathrm{L})$ and arg-vasopressin $(0.5 \mathrm{pmol} /$ $\mu \mathrm{L}$ ), was directly infused at a rate of $15 \mu \mathrm{L} / \mathrm{min}$ and ionized by positive-mode ESI (Figure 2a-d). The mixture results in singly protonated ketoconazole at $\mathrm{m} / \mathrm{z} 531.3$, and doubly protonated arg-vasopressin at $\mathrm{m} / \mathrm{z}$ 542.8. Protonated ketoconazole and doubly protonated arg-vasopressin were filled sequentially, each under unit mass resolution, into the LIT and then

Table 1. CVs and accuracies in analyzing triply protonated angiotensin Iat $m / z 432.9$ using SRM and tEMC

\begin{tabular}{|c|c|c|c|c|}
\hline & Actual Concentration $(\mathrm{fmol} / \mu \mathrm{L})$ & Calculated Concentration $(\mathrm{fmol} / \mu \mathrm{L})$ & $\mathrm{CV}(\%)$ & Accuracy $(\%)$ \\
\hline \multirow[t]{5}{*}{ SRM: $m / z 432.9$ to $m / z 619.8$} & 0.6 & 0.9 & 0 & 147 \\
\hline & 1.2 & 0.7 & 45 & 55 \\
\hline & 4.9 & 5.6 & 11 & 113 \\
\hline & 9.8 & 11 & 14 & 114 \\
\hline & 39.1 & 43 & 2 & 110 \\
\hline \multirow[t]{5}{*}{ SRM: $m / z 432.9$ to $m / z 647.5$} & 0.6 & 0.4 & 28 & 71 \\
\hline & 1.2 & 1.1 & 18 & 94 \\
\hline & 4.9 & 5.4 & 6 & 110 \\
\hline & 9.8 & 10.1 & 2 & 104 \\
\hline & 39.1 & 41.4 & 1 & 107 \\
\hline \multirow[t]{5}{*}{ SRM: Sum of the two transitions } & 0.6 & 0.7 & 16 & 110 \\
\hline & 1.2 & 1.0 & 13 & 84 \\
\hline & 4.9 & 4.6 & 9 & 93 \\
\hline & 9.8 & 8.8 & 6 & 91 \\
\hline & 39.1 & 44.5 & 4 & 114 \\
\hline \multirow[t]{5}{*}{ tEMC } & 0.6 & 0.6 & 5 & 92 \\
\hline & 1.2 & 1.2 & 13 & 100 \\
\hline & 4.9 & 5.8 & 1 & 118 \\
\hline & 9.8 & 10.9 & 4 & 112 \\
\hline & 39.1 & 44.3 & 3 & 113 \\
\hline
\end{tabular}



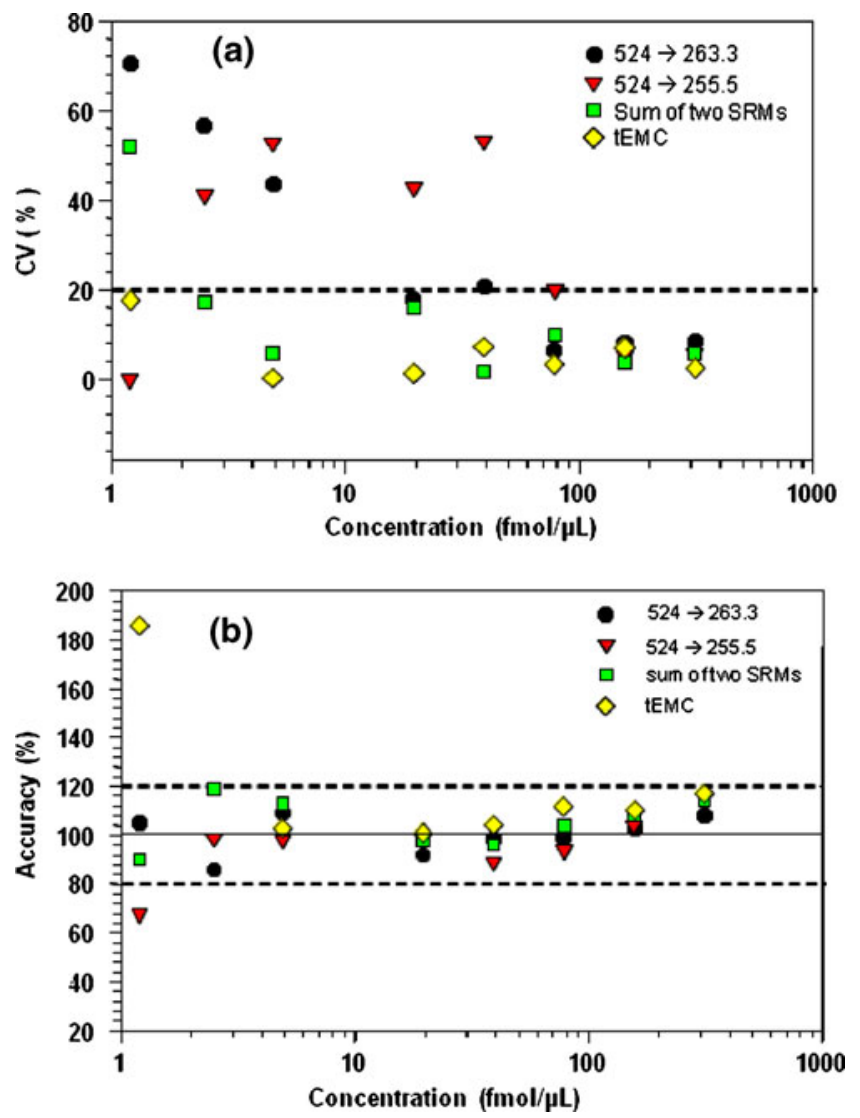

Figure 4. Analysis of doubly protonated angiotensin II: (a) $\mathrm{CV}$ and (b) accuracy as a function of concentration. Black circles, SRM transition $\mathrm{m} / \mathrm{z} 523.8$ to 263.3; red triangles, SRM transition $\mathrm{m} / \mathrm{z} 523.8$ to 255.5 ; green squares, SRM summing the two transitions; and yellow diamonds, tEMC

subjected to charge discrimination by controlling the EXB voltage prior to mass analysis. This resulted in changes to both the absolute and relative abundances of the two differently charged ions. The changes from an EXB voltage of 0 to $2.7 \mathrm{~V}$ are plotted in Figure 2d, which shows no ions were retained in the LIT when the barrier was $0.5 \mathrm{~V}$ or less. Trapping of doubly protonated arg-vasopressin and singly protonated ketoconazole in the LIT began with an EXB voltage $\geq 0.6 \mathrm{~V}$ and $1.1 \mathrm{~V}$, respectively. For optimal discrimination between singly and doubly charged ions, it is apparent that an EXB voltage of $1.1 \mathrm{~V}$ should be employed. At a barrier of $1.1 \mathrm{~V}$, only a trace of singly protonated ketoconazole was retained by the LIT versus $\sim 30 \%$ of doubly protonated arg-vasopressin (Figure $2 \mathrm{a}$ and d). At a higher EXB voltage of $1.5 \mathrm{~V}, \sim 35 \%$ of protonated ketoconazole and over $90 \%$ of doubly protonated argvasopressin were retained (Figure $2 \mathrm{~b}$ and $\mathrm{d}$ ); discrimination between the two ions was less effective. The ketoconazole and arg-vasopressin ions have different $m / z$ ratios and can arguably be more conveniently discriminated by means of mass filtering. Although retaining only $\sim 30 \%$ of the multiply charged ions may be viewed as a significant loss of the original signal, this is offset by over $90 \%$ loss of the chemical interference; in addition, this level of performance appeared to be reproducible among all peptides; thus, making it predictable and reproducible across multiple analytes. This behavior is different from SRM which exhibits high variability in transmission and fragmentation efficiencies among peptides and charge states.

A second mixture that simulated isobaric interference by protonated ketoconazole on doubly protonated bradykinin at $\mathrm{m} / \mathrm{z} 530.8$ was also evaluated (Figure $2 \mathrm{e}-\mathrm{h}$ ). The two ions are unresolved when $\mathrm{Q} 1$ is operated at unit mass resolution, and both were transmitted into the LIT under typical analytical conditions. At an EXB voltage of $1.1 \mathrm{~V}$, only doubly protonated bradykinin was retained by the LIT at an abundance of $7.0 \times 10^{4} \mathrm{cps}$ (Figure 2e) despite transmission of five times more abundant protonated ketoconazole (assuming that the response per ion was identical). At an EXB voltage higher than $1.1 \mathrm{~V}$, some trapping of singly protonated ketoconazole ensued: Figure $2 \mathrm{f}$ and $\mathrm{g}$ shows tEMC mass spectra at EXB voltages of 1.5 and $2.0 \mathrm{~V}$, respectively. Under these conditions, protonated ketoconazole dominated the mass spectra, which is apparent in a comparison with theoretical isotopic distributions of protonated ketoconazole and doubly protonated bradykinin at a ratio of 5:1 (Figure 2g). Evidently, results from the analyses of the two mixtures show that an EXB voltage of $1.1 \mathrm{~V}$ provides optimal discrimination against singly charged

Table 2. LLOQs of six multiply protonated peptide ions in dilute horse plasma acquired using the optimal single SRM transition, sum of SRM transitions, and tEMC

\begin{tabular}{|c|c|c|c|}
\hline \multirow[b]{2}{*}{ Peptide } & \multicolumn{3}{|c|}{ LLOQ (fmol/ $\mu \mathrm{L}[\mathrm{ng} / \mathrm{mL}])$} \\
\hline & SRM Single Transition & SRM Summing ${ }^{\mathrm{b}}$ & tEMC \\
\hline$(\text { Arg-vasopressin }+2 \mathrm{H})^{2+}(542.7)^{\mathrm{a}}$ & $9.8[10.6]$ & $4.9[5.3]$ & $2.5[2.7]$ \\
\hline$(\text { Angiotensin } \mathrm{II}+2 \mathrm{H})^{2+}(523.8)$ & $19.5[20.7]$ & $2.5[2.6]$ & $4.9[5.1]$ \\
\hline$(\text { Angiotensin } \mathrm{I}+3 \mathrm{H})^{3+}(432.9)$ & $0.61[0.79]$ & $0.61[0.79]$ & $0.61[0.79]$ \\
\hline$(\text { Melittin }+3 \mathrm{H})^{3+}(949.6)$ & $19.5[55.5]$ & $19.5[55.5]$ & $9.8[27.9]$ \\
\hline$(\text { Melittin }+4 H)^{4+}(712.7)$ & $39.1[111.2]$ & $19.5[55.5]$ & $39.1[111.2]$ \\
\hline$(\text { Exenatide }+4 \mathrm{H})^{4+}(1047.5)$ & $11.6[48.6]$ & $11.6[48.6]$ & $11.6[48.6]$ \\
\hline$(\text { Arg-insulin }+6 \mathrm{H})^{6+}(995.0)$ & $9.8[58.5]$ & $9.8[58.5]$ & $9.8[58.5]$ \\
\hline
\end{tabular}

a $m / z$ value, barg-vasopressin: 542.7 to $328.6,535.2$, and 921.7 ; angiotensin II: 523.8 to 263.3 and 255.5 ; angiotensin I: 432.9 to 619.8 and 647.5 ; melittin: 949.6 to $1,106.7,1,056.2$, and $494.3 ; 712.7$ to $894.5,813.5$, and 542.9 ; exenatide: $1,047.5$ to 397.5 and 300.0 ; arg-insulin: 995.0 to $1,161.0$ and $1,138.0$ 
species in tEMC analyses of doubly and other multiply protonated peptides (vide infra).

\section{Comparisons of SIM, SRM, and tEMC}

A sample of 12.3 fmol angiotensin I dissolved in $10 \mu \mathrm{L}$ dilute horse plasma was injected on column and used to evaluate the performance of SIM, SRM, and tEMC under LC-MS and LC-MS/MS conditions, where appropriate (Figure 3). SIM failed to detect triply protonated angiotensin I $(\mathrm{m} / \mathrm{z} 432.9)$ among a steadily increasing baseline resulting from singly charged background ions (Figure 3a). By contrast, SRM of two transitions $(\mathrm{m} / \mathrm{z} 432.9$ dissociating to $\mathrm{m} / \mathrm{z} 619.8$ and 647.5) detected angiotensin I at a retention time of $4.19 \mathrm{~min}$ (Figure 3b), as did tEMC (Figure 3c). While the SRM method showed less interference than the other two detection modes, SRM is typically associated with a very significant decrease in ion abundance (only $1.2 \%$ of ions are retained from MS to MS/MS for angiotensin I, and 0.3 to $5 \%$ for other peptides studied). Despite being less selective than SRM, tEMC provided adequate resolution and discrimination against background ions (see the red trace showing analysis of diluted horse plasma, the biological matrix in this experiment); in addition, optimization in tEMC was much simpler than that in SRM.

Calibration plots of doubly protonated arg-vasopression performed in triplicate analyses using SRM (summing of three transitions: $\mathrm{m} / \mathrm{z} 542.7$ to $\mathrm{m} / \mathrm{z} 921.7,535.2$, and 328.6) and using tEMC were linear with coefficients of determination $\left(r^{2}\right)$ of 0.9948 and 0.9923 , respectively. Comparable performance was also evident for other peptide ions. These ions include doubly protonated angiotensin II: SRM (summing of two transitions: $\mathrm{m} / \mathrm{z} 523.8$ to $\mathrm{m} / \mathrm{z} 263.3$ and 255.5), $r^{2}=0.991$, and tEMC, $r^{2}=0.985$; quadruply protonated melittin: SRM (summing three transitions: $\mathrm{m} / \mathrm{z} 712.7$ to $\mathrm{m} / \mathrm{z}$ 894.7, 813.5, and 542.9), $r^{2}=0.995$, and tEMC, $r^{2}=0.974$; triply protonated melittin: SRM (summing three transitions: $\mathrm{m} / \mathrm{z} 949.6$ to $\mathrm{m} / \mathrm{z}$ 1,106.7, 1,056.2, and 494.3), $r^{2}=0.9996$, and tEMC, $r^{2}=$ 0.9990; quadruply protonated exenatide: SRM (summing two transitions: $\mathrm{m} / \mathrm{z} 1,047.5$ to $\mathrm{m} / \mathrm{z} 397.5$ and 300.0$), r^{2}=0.989$, and tEMC, $r^{2}=0.980$; and hexaprotonated (6+) arg-insulin: SRM (summing two transitions: $\mathrm{m} / \mathrm{z} 995.0$ to $\mathrm{m} / \mathrm{z} \quad 1,138.0$ and 1,161.0), $r^{2}=0.995$, and tEMC, $r^{2}=0.988$. Details can be found in the Supplementary Information section.

Additionally, details of the analysis of triply protonated angiotensin I $(\mathrm{m} / z$ 432.9) are used to further illustrate the comparable performance of SRM and tEMC (Table 1). For SRM, accuracies and precisions (CV) in terms of two transitions, $\mathrm{m} / \mathrm{z} 432.9$ to 619.8 and 647.5 , are shown individually and after summing. Evidently, the performance of SRM and tEMC is comparable. Figure 4 shows a comparison on doubly protonated angiotensin II in a graphical format. For SRM, the CVs and accuracies for transitions from $\mathrm{m} / \mathrm{z} 523.8$ to $\mathrm{m} / \mathrm{z} 263.3$ and 255.5 are plotted individually and after summing. The accuracies between SRM and tEMC are similar and do not deviate by more than
$20 \%$, except for the sample with the lowest concentration. tEMC appears to show better precision than SRM, especially for samples of lower concentrations. The lower limits of quantification for seven multiply protonated peptides as determined by using SRM (single transition and summed transition) and tEMC are given in Table 2. As a general trend, tEMC provided LLOQs lower than or similar to those of SRM when single transitions were considered. It was possible to improve the LLOQ in some cases when two SRM transitions were summed, but overall the LLOQs were comparable between tEMC and summed SRM. Although tEMC does not provide a systematic improvement in detection limits, its major advantage lies in the simplicity of optimization and instrumental control (only a minor tuning of EXB is required). Conservatively, we deem tEMC to perform just as well as SRM in terms of accuracy and precision, after taking into consideration of all results.

Future research in tEMC will involve evaluating its performance for the quantification of proteins or large peptides of even higher charge states, as well as implementing a 'dualfill' tEMC experiment, wherein two or more separate charge states from a single peptide/protein (e.g., $[\text { melittin }+3 \mathrm{H}]^{3+}$ at $\mathrm{m} / \mathrm{z} 949.6$ and $[\text { melittin }+4 \mathrm{H}]^{4+}$ at $\mathrm{m} / \mathrm{z}$ 712.7) can be introduced, trapped, and manipulated in the LIT.

\section{Conclusion}

In the tEMC mode of operation, peptides are transmitted into the LIT without fragmentation and are ultimately detected as intact species after a charge-separation step. This approach enables selective detection of multiply charged peptides with LLOQs that are comparable to SRM but with much less optimization and tuning required by the user. Indeed, tEMC requires a onetime determination of the optimal EXB voltage and this value can be used across multiple peptides of differing charge states, as the primary discrimination is against background ions which tend to be singly charged. Compared to SRM, which requires pre-determination of fragment ions and associated collision energies, tEMC is by far simpler and universal. tEMC enables determination of multiply charged therapeutic peptides with minimal instrument tuning, making it a convenient, yet sensitive and selective tool for supporting the discovery and development of new therapeutic peptide candidates.

\section{Acknowledgments}

This work was supported by the Natural Sciences and Engineering Research Council (NSERC) of Canada and AB SCIEX. We thank Olga Krakovska for discussions regarding statistical analysis of the data.

\section{References}

1. John, H.: Liquid Chromatography-Mass Spectrometry in Peptide Drug Discovery and Development. Anal. Bioanal. Chem. 381, 51-53 (2005)

2. Szymkowski, D.E.: Rational, Optimization of Proteins as Drugs: A New Era of 'Medicinal Biology'. Drug Disc. Today 9, 381-383 (2004) 
3. Crommelin, D.J.A., Storm, G., Verrijk, R., de Leede, L., Jiskoot, W., Hennink, W.E.: Shifting Paradigms: Biopharmaceuticals versus Low Molecular Weight Drugs. Int. J. Pharm. 266, 3-16 (2003)

4. Marx, V.: Watching Peptide Drugs Grow Up. Chem. Eng. News 83, 1724 (2005)

5. Kirchherr, H.: Data Evaluation in LC-MS. In Quantification in LC and $G C$, Kuss, H.J.; Kromidas, S., Eds; Wiley-VCH: Weinheim, 2009; pp. 243-259

6. Anderson, L., Hunter, C.L.: Quantitative Mass Spectrometric MRM Assays for Major Plasma Proteins. Mol. Cell. Proteomics 5, 573-588 (2006)

7. Xia, S.F., Zhang, L., Qiu, B., Lu, M.H., Chi, Y.W., Chen, G.N.: OnLine Preconcentration and Quantitative Analysis of Peptide Hormone of Brain and Intestine Using On-Column Transient Isotachophoresis Coupled with Capillary Electrophoresis/Electrospray Ionization Mass Spectrometry. Rapid Commun. Mass Spectrom. 22, 3719-3726 (2008)

8. Berna, M., Schmalz, C., Duffin, K., Mitchell, P., Chambers, M., Ackermann, B.: Online Immunoaffnity Liquid Chromatography/Tandem Mass Spectrometry Determination of a Type II Collagen Peptide Biomarker in Rat Urine: Investigation of the Impact of CollisionInduced Dissociation Fluctuation on Peptide Quantitation. Anal. Biochem. 356, 235-243 (2006)

9. Aebersold, R., Goodlett, D.R.: Mass Spectrometry in Proteomics. Chem. Rev. 101, 269-295 (2001)

10. Frank, A.M.A.: Ranking-Based Scoring Function for Peptide-Spectrum Matches. J. Proteome Res. 8, 2226-2240 (2009)

11. Kaufmann, A., Butcher, P., Maden, K., Widmer, M., Giles, K., Uria, D. Are Liquid Chromatography/Electrospray Tandem Quadrupole Fragmentation Ratios Unequivocal Confirmation Criteria? Rapid Commun. Mass Spectrom. 23, 985-998 (2009)

12. Wolf, R.; Roche, F.; Hoffmann, T.; Demuth, H.-U.: Immunoprecipitation and Liquid Chromatographic-Mass Spectrometric Determination of the Peptide Glucose-Dependent Insulinotropic Polypeptides GIP1-42 and GIP3-42 from Human Plasma Samples. New Sensitive Method to Analyze Physiological Concentrations of Peptide Hormones. J. Chromatogr. A 926, 21-27 (2001)

13. Guevremont, R., Purves, R.W.: Atmospheric Pressure Ion Focusing in a High-Field Asymmetric Waveform Ion Mobility Spectrometer. Rev. Sci. Instrum. 70, 1370-1383 (1999)

14. Shvartsburg, A.A. Differential Ion Mobility Spectrometry: Nonlinear Ion Transport and Fundamentals of FAIMS, CRC Press, Boca Raton, FL, 2009, pp 1-299

15. Valentine, S.J., Kulchania, M., Srebalus Barnes, C.A., Clemmer, D.E.: Multidimensional Separations of Complex Peptide Mixtures: a Combined High-Performance Liquid Chromatography/Ion Mobility/Time-of-Flight Mass Spectrometry Approach. Int. J. Mass Spectrom. 212, 97-109 (2001)
16. Barnett, D.A., Ells, B., Guevremont, R., Purves, R.W.: Application of ESI-FAIMS-MS to the Analysis of Tryptic Peptides. J. Am. Soc. Mass Spectrom. 13, 1282-1291 (2002)

17. Kindy, J.M., Taraszka, J.A., Regnier, F., Clemmer, D.E.: Quantifying Peptides in Isotopically Labeled Protease Digests by Ion Mobility/Timeof-Flight Mass Spectrometry. Anal. Chem. 74, 950-958 (2002)

18. Levin, D.S., Miller, R.A., Nazarov, E.G., Vouros, P.: Rapid Separation and Quantitative Analysis of Peptides Using a New NanoelectrosprayDifferential Mobility Spectrometer-Mass Spectrometer System. Anal. Chem. 78, 5443-5452 (2006)

19. Xia, Y.-Q., Wu, S.T., Jemal, M.: LC-FAIMS-MS/MS for Quantification of a Peptide in Plasma and Evaluation of FAIMS Global Selectivity from Plasma Components. Anal. Chem. 80, 7137-7143 (2008)

20. Klaassen, T., Szwandt, S., Kapron, J.T., Roemer, A.: Validated Quantitation Method for a Peptide in Rat Serum Using Liquid Chromatography/High-Field Asymmetric Waveform Ion Mobility Spectrometry. Rapid Commun. Mass Spectrom. 23, 2301-2306 (2009)

21. Chernushevich, I.V., Fell, L.M., Bloomfield, N., Metalnikov, P.S., Loboda, A.V.: Charge State Separation for Protein Applications Using a Quadrupole Time-of-Flight Mass Spectrometer. Rapid Commun. Mass Spectrom. 17, 1416-1424 (2003)

22. Le Blanc, J.C.Y., Hager, J.W., Ilisiu, A.M.P., Hunter, C., Zhong, F., Chu, I.: Unique Scanning Capabilities of a New Hybrid Linear Ion Trap Mass Spectrometer (QTRAP) Used for High Sensitivity Proteomics Applications. Proteomics 3, 859-869 (2003)

23. Hager, J. W.: Method of Mass Spectrometry to Enhance Separation of Ions with Different Charges. U.S. Patent Application 20040183005

24. Drogaris, P., Le Blanc, J.C.Y., Fitzgerald, J.E., Lowndes, N.F., Verreault, A., Thibault, P.: Enhanced Protein Detection Using a Trapping Mode on a Hybrid Quadrupole Linear Ion Trap (Q-Trap). Anal. Chem. 81, 6300-6309 (2009)

25. Le Blanc, J.C.Y.; Hao, C.; Campbell, J.L.; Siu, K.W.M.: Using Targeted Charge Separation to Improve Detection Limits in Therapeutic Peptide Quantitation. Proceedings of the $57^{\text {th }}$ Annual American Society for Mass Spectrometry Conference on Mass Spectrometry and Allied Topics, Philadelphia, PA, June 2009

26. Federal Drug Administration, Guidance for Industry: Bioanalytical Method Validation, May 2001, http://www.fda.gov/downloads/Drugs/ GuidanceComplianceRegulatoryInformation/Guidances/UCM070107. pdf, accessed on May 7, 2010

27. Introduction to Modern Liquid Chromatography, Snyder, L.R; Kirkland, J.J.; Dolan, J.W., Eds.; Wiley \& Sons Inc: Weinheim, 2010; Chapter 11

28. Londry, F.A., Hager, J.W.: Mass Selective Axial Ion Ejection from a Linear Quadrupole Ion Trap. J. Am. Soc. Mass Spectrom. 14, 11301147 (2003) 